\title{
Approach Thermal Habitat Assessment in N’Djamena in Chad
}

\author{
Ahmat Charfadine1, Mahamat Barka1 ${ }^{*}$, Abakar Mahamat Tahir1, \\ Mohagir Ahmed Mohammed1, Pr. Salif Gaye ${ }^{2}$ \\ ${ }^{1}$ Laboratory of Renewable Energy and Materials Premises of the University of N'Djamena, N'Djamena, Chad \\ ${ }^{2}$ Laboratory of Materials, Mechanics and Hydraulics of the University of Thies, Thies, Senegal \\ Email: *abakarmt@gmail.com
}

Received 17 November 2015; accepted 12 February 2016; published 16 February 2016

Copyright (C) 2016 by authors and Scientific Research Publishing Inc.

This work is licensed under the Creative Commons Attribution International License (CC BY).

http://creativecommons.org/licenses/by/4.0/

(c) () Open Access

\begin{abstract}
For the earth material construction is the most used one in Chad, the object of this work is the assessment of the thermal quality of earthen adobe mixed with straw. Different simulation software analyzes the CoDyBa which is conducted to determine the behavior of this material compared to living comfort thanks to the Fanger model, which is a method of approach to thermal comfort standpoint. The study focused on two configurations on the formation of the walls of a room. The first of these configurations led to the definition of a first cell constructed earthen adobe named Batter roofing sheet aluminum and a second cell built in blocks named Batbet which had the same geometric characteristics (thickness, dimensions and side openings) and covered in the same manner as the ground cell. The different comfort indices PMV and PPD values of these two configurations of habitat were identified and were used to determine their thermal comfort rating.
\end{abstract}

\section{Keywords}

Habitat Assessment, Habitat Suitability, Building Monitoring, Climate Change, Thermal Habitat, Orientation, Solar

\section{Introduction}

Thermal comfort is not only a function of the individual but also depends on its habitat. Thus we will study the factors related to the individual, his vesture and activity, then the thermal qualities of its habitat. A building is never steady because:

- The outside temperature is variable;

*Corresponding author.

How to cite this paper: Charfadine, A., Barka, M., Tahir, A.M., Mohammed, M.A. and Gaye, Pr.S. (2016) Approach Thermal Habitat Assessment in N'Djamena in Chad. World Journal of Engineering and Technology, 4, 82-102.

http://dx.doi.org/10.4236/wjet.2016.41009 
- Solar radiation, periodic, may be picked up by some opaque parts of envelopes or may enter directly inside through the openings;

- The establishment of a thermal power inside the building due to its operation (metabolism occupants, appliances, cooking...) is variable in time;

- The building is ventilated with an air flow (possibly variable temperature gradient variable between inside and outside).

The problem is dynamic and relatively complex, that is why simulations are necessary, through the IT tool to see the thermal behavior of walls of a social housing in a given area. Climate databases needed to run the software in use (CoDyBa) consist of information about schedules:

- The air temperature outside $\left(T_{a}\right)$;

- Global solar flux received by a horizontal plane;

- The height of the sun in relation to the south;

- The relative humidity of the outside air.

So we built a base of climatic data from the meteorological data from Chad (ten-year average) [1].

\section{Formatting Climate Data}

Comparisons inter weather stations and global climate data processing of Chad helped define homogeneous climatic zones. To this end the influence of latitude and altitude on weather data [1] was analyzed and defined, homogeneous areas were checked in terms of humidity, rainfall and regimes the wind. So there are three climate zones [2]:

- Climate zone A: Tropical climate;

- Climatic zone B: Sahelian climate;

- Climatic zone C: Saharan climate.

As part of this study, we will choose the city of N’Djamena as "sample city” representing the climate zone B.

\subsection{Meteorological Database for the Bioclimatic Design of the Habitat}

To evaluate the thermal comfort conditions in housing, it is necessary to be in the most adverse weather conditions, i.e. when the heat input is maximum [3] [4] Thus the weather data will be determined for The hottest time of the year in the dry season for the selected climate zone. For this assessment, we will use the thermal simulation software CoDyBa buildings, which uses meteorological databases hours and hours sorting the site (temperature, humidity and sunlight, wind, ...). In the absence of such information in local climatological files, it is possible to reconstruct the typical days from the average maximum and minimum averages of the meteorological data of the place.

\subsubsection{Outdoor Temperature Dry and the Relative Humidity}

The objective of bioclimatic architecture is to reproduce comfort conditions identical to those experienced by an individual on the outside, in the shade (under a tree), with a light breeze [3] [5] [6]. For this, the average climatic characteristics of a region (in temperature and humidity) are to be implemented in non-conditioned spaces. Table 1 presents the most favorable climatic characteristics which could result in non-conditioned spaces. Table 2 provides the basic external conditions in the dry season and cool season by noting:

- $T_{x}$, the maximum temperature;

- $T_{n}$, the minimum temperature;

- $T_{m}$, the average temperature;

- $H_{\text {moy, }}$ the average relative humidity.

\subsubsection{Ventilation}

Ventilation is the most important parameter in evaluating the humidity in the comfort [7] [8] building is the key driver. To this end, a house may have satisfactory thermal performance if it is pointing aerodynamically in the wind direction. Table 3 provides for zone B average speed and direction of prevailing winds.

\subsubsection{Rainfall}

If rainfall does not directly climate comfort, it is against directly related to the sustainability of buildings; and 
Table 1. Bases of external conditions for the climate zone B.

\begin{tabular}{ccccccccccccc}
\hline & Jan. & Feb. & March & April & May & June & July & August & Sept. & Oct. & Nov. & Dec. \\
\hline$T_{x}\left({ }^{\circ} \mathrm{C}\right)$ & 31.9 & 33.6 & 39 & 41.1 & 40 & 38 & 34.1 & 31.9 & 34.1 & 37.6 & 36.6 & 32.6 \\
$T_{n}\left({ }^{\circ} \mathrm{C}\right)$ & 14.9 & 17.4 & 22.7 & 25.6 & 26.4 & 25.3 & 23.5 & 22.8 & 23.1 & 22.4 & 19.5 & 15.6 \\
$T_{m}\left({ }^{\circ} \mathrm{C}\right)$ & 23.4 & 25.5 & 30.9 & 33.4 & 33.2 & 31.7 & 28.8 & 27.4 & 28.6 & 30 & 28.1 & 24.1 \\
$H_{\text {moy }}(\%)$ & 22.1 & 18.5 & 18.4 & 22.4 & 39.6 & 50.3 & 67.6 & 72.5 & 66.3 & 43.3 & 25.9 & 22.6 \\
\hline
\end{tabular}

Table 2. Basic external conditions dry and cool season (zone B).

\begin{tabular}{ccccc}
\hline & \multicolumn{2}{c}{ External condition base (dry season) } & \multicolumn{2}{c}{ Basic external condition (cool season) } \\
\hline Zone & Temperatures max dry. $\left({ }^{\circ} \mathrm{C}\right)$ & Warmest month & Temperature mini dry. $\left({ }^{\circ} \mathrm{C}\right)$ & Cooler month \\
\hline B (N’Djamena) & 41.1 & April & 14.9 & January \\
\hline
\end{tabular}

Table 3. Climatic characteristics (wind) and geographical zone B.

\begin{tabular}{cccccccc}
\hline \multicolumn{9}{c}{ Prevailing winds } & \multicolumn{2}{c}{ Wind Speed } \\
\hline Zone & Dry season & Rain season & Dry season & Rain season & Latitude & Longitude & Elevation \\
\hline B (N’Djaména) & NNE & SSW & $3.2 \mathrm{~m} / \mathrm{s}$ & $2.4 \mathrm{~m} / \mathrm{s}$ & $12^{\circ} 07 \mathrm{~N}$ & $15^{\circ} 03 \mathrm{E}$ & $298 \mathrm{~m}$ \\
\hline
\end{tabular}

the works must be protected against the rain. Table 4 shows the monthly average amount of precipitation in the area B.

\subsubsection{Sunshine}

Solar radiation plays an important role in thermal exchanges of bioclimatic habitat because it may represent an important, sometimes the most important, external heat gains of the building.

The heat loads in the building due to solar radiation can be reduced by the shadows cast by the projections, canopies and neighboring buildings, the construction design for natural cooling. To determine these shadows, we must know the position of the sun: its height and azimuth. Table 5 and Table 6 give the monthly average of the average insulation and global and direct radiation on a horizontal plane.

\subsubsection{Selection of Typical Days}

Given Chad's climate data available, we have selected for the climate zone B sunny days during the hottest months of the dry season and we calculated hour by hour $(24 \mathrm{~h})$ the average of the various parameters: temperature, relative humidity, global flows and diffuse flux, speed and direction of wind, which allowed the establishment of the day type of climatic zone B, a typical day for the month of April (Figure 1).

Table 7 relates to the hottest month (April) in the year of parameter values for calculating the flux stream received in the city of Ndjamena [2] [9].

\subsubsection{Solar Radiation Incident}

Moreover, in Table 8, schedules climate files on a full year have been exploited to calculate the maximum solar global radiation incident solar radiation average monthly and annual incident on the various plans for area B [2] [9].

\subsection{Definition of Local Types}

Local types are defined according to current construction practices in Chad. We chose local surfaces, types of walls and dimensions of current openings.

The selected configuration of the local consists of a bedroom and a separate living room by a partition. The 
Table 4. Monthly average height in meters (mm).

\begin{tabular}{cccccccccccccc}
\hline \multicolumn{10}{c}{ Zone B } \\
Month & Jan & Feb. & March & April & May & June & July. & August & Sept. & Oct. & Nov. & Dec. \\
\hline H (mm) & 0 & 0 & 0 & 8.40 & 31.36 & 38.72 & 141.94 & 156.33 & 73.14 & 14.64 & 0 \\
\hline
\end{tabular}

Table 5. Average daily sunshine hours.

\begin{tabular}{cccccccccccccc}
\hline Zone & Jan. & Feb. & March & April & May & June & July & August & Sept. & Oct. & Nov. & Dec. \\
\hline B & 9.5 & 9.4 & 8.8 & 8.8 & 8.7 & 8.3 & 7.1 & 6.7 & 7.7 & 9.1 & 9.8 & 9.3 \\
\hline
\end{tabular}

Table 6. Average monthly global measured and direct radiation on a horizontal plane.

\begin{tabular}{cccccccccccccc}
\hline Zone & Flow Wh/d & Jan. & Feb. & March & April & May & June & July & August & Sept. & Oct. & Nov. & Dec. \\
\hline \multirow{2}{*}{ B } & IRg & 4419 & 4976 & 6549 & 7236 & 7323 & 6641 & 5193 & 5075 & 5135 & 5212 & 5090 & 4121 \\
& IRd & 3058 & 3493 & 4821 & 5450 & 5510 & 4855 & 3414 & 3276 & 3634 & 4137 & 3664 & 2764 \\
\hline
\end{tabular}

Table 7. Parameter values used for the constitution of a typical day.

\begin{tabular}{cccccccc}
\hline Zone & Month & $T_{x}$ at $15 \mathrm{pm}$ & $T_{n}$ at 3 am & Latitude & Transparency Coefficient & Specific Humidity & Insolation Fraction \\
\hline N’Djaména & April & $41.2^{\circ} \mathrm{C}$ & $25.6^{\circ} \mathrm{C}$ & $12^{\circ} 07$ & 0.75 & 0.79 & 0.78 \\
\hline
\end{tabular}

* Insolation Fraction: average insolation report on the maximum duration of one day. ${ }^{*}$ Transparency Coefficient: coefficient to calculate the live stream received by a horizontal plane sky "clear" (no clouds).

Table 8. Global solar radiation incident on the various plans (average in $24 \mathrm{~h}$ ).

\begin{tabular}{cccc}
\hline & \multicolumn{3}{c}{ Zone B } \\
\hline & G monthly average (the hottest month) in $\mathbf{~} / \mathbf{m}^{2}$ & G annual average in $\mathbf{W} / \mathbf{m}^{2}$ & 78 \\
North & 68 & 149 & 166 \\
East & 161 & 126 & 218 \\
South & 65 & 149 \\
Ouest & 161 & 238 \\
\hline
\end{tabular}

local is oriented along the east-west longitudinal axis.

\subsubsection{Color Walls}

We note that all exterior walls are light colored whose absorption coefficient is 0.30 (white). The absorption coefficient of the inner walls is 0.35 (beige).

\subsubsection{Breakdown}

Natural ventilation is ensured local with a fresh air flow that is ten times the volume of the space concerned (10 $\mathrm{V} / \mathrm{h}$ ) and a medium speed cited above.

\subsubsection{Description of Premises}

The dimensions and two selected local characteristics are as follows:

*Room

Unconditioned local presentation on the two adjacent walls to the east and south.

- Total floor space: $3.5 \mathrm{~m} \times 3.0 \mathrm{~m}=10.5 \mathrm{~m}^{2}$

- Height: $3.40 \mathrm{~m}$

- Height under false ceiling: $2.60 \mathrm{~m}$

- Volume: $10.5 \mathrm{~m}^{2} \times 2.60 \mathrm{~m}=27.3 \mathrm{~m}^{3}$

- Air exchange rate: $273 \mathrm{~m}^{3}$ 


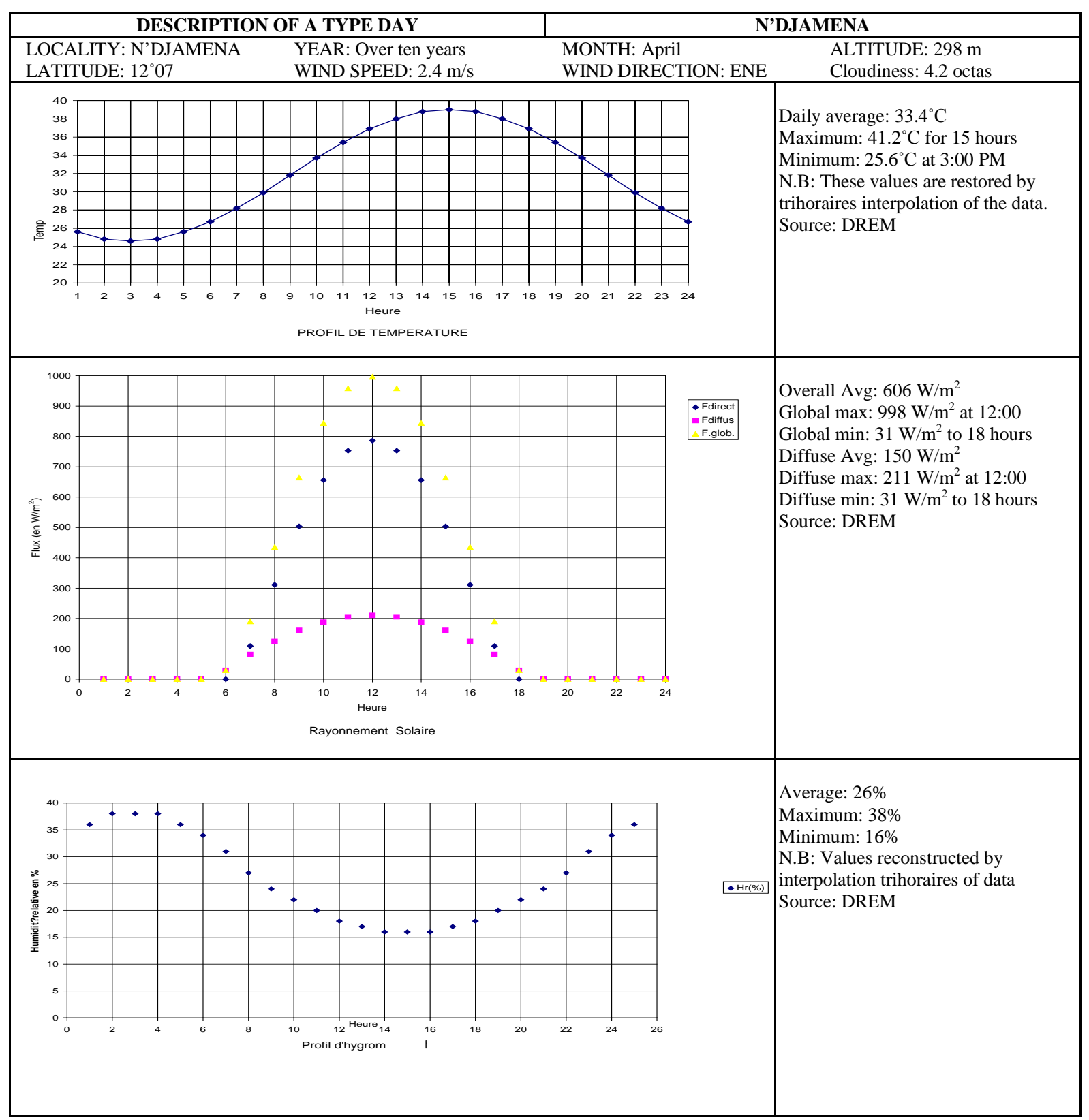

Figure 1. Description of a typical day of N'Djamena (month of April).

*Openings:

North Window of $1.20 \mathrm{~m} \times 1 \mathrm{~m}$

Clear glass transmission coefficient $\mathrm{K}=5.8 \mathrm{~W} / \mathrm{m}^{2} /{ }^{\circ} \mathrm{C}$.

South Window of $1.20 \mathrm{~m} \times 1 \mathrm{~m}$

Clear glass transmission coefficient $\mathrm{K}=5.8 \mathrm{~W} / \mathrm{m}^{2} /{ }^{\circ} \mathrm{C}$.

*Features walls:

- Exterior wall: ground brick $22 \mathrm{~cm}$ plus $1 \mathrm{~cm}$ and $2 \mathrm{~cm}$ ground respectively coated on both interior and exterior siding;

- Interior Partition: clay brick $22 \mathrm{~cm}+1.5 \mathrm{~cm}$ of earth coated on both facings;

- Low Floor: $10 \mathrm{~cm}$ of heavy concrete.

- Roof: made of sheet metal, with an air gap of an average thickness of $40 \mathrm{~cm}$ and ceiling.

Time profiles of natural ventilation of the space concerned are presented in Table 9 . 
Table 9. Time profiles of natural ventilation of premises.

\begin{tabular}{cccccccc}
\hline \multicolumn{7}{c}{ ROOM (maximum flow: $273 \mathrm{~m}^{3}$ ) } \\
\hline \multicolumn{7}{c}{ Profile week } & \multicolumn{5}{c}{ Profile weekend } \\
\hline Time & Flow \% & Time & Flow \% & Time & Flow \% & Time & Flow \% \\
\hline 1 & 100 & 13 & 0 & 1 & 100 & 13 & 0 \\
2 & 100 & 14 & 0 & 2 & 100 & 14 & 50 \\
3 & 100 & 15 & 0 & 3 & 100 & 15 & 50 \\
4 & 100 & 16 & 0 & 4 & 100 & 16 & 50 \\
5 & 100 & 17 & 50 & 5 & 100 & 17 & 50 \\
6 & 100 & 18 & 50 & 6 & 100 & 18 & 50 \\
7 & 50 & 19 & 100 & 7 & 0 & 19 & 100 \\
8 & 50 & 20 & 100 & 8 & 0 & 20 & 100 \\
9 & 50 & 21 & 100 & 9 & 0 & 21 & 100 \\
10 & 50 & 22 & 100 & 10 & 0 & 22 & 100 \\
11 & 50 & 23 & 100 & 11 & 0 & 23 & 100 \\
12 & 0 & 24 & 100 & 12 & 0 & 24 & 100 \\
\hline
\end{tabular}

To check the quality of the thermal comfort of our home land, we compared it to another habitat with the same construction plans but an envelope with cinderblock cement plaster.

\section{Simulation}

For the simulation, so we considered two configurations on the formation of the walls of this room. The first of these configurations results in the definition of a first cell in ground named BATTER roofing sheet aluminum and a second concrete cell named BATBET which has the same geometrical characteristics (thickness, lateral dimensions and openings) and covered with the same so that the earth cell.

In both configurations, the main facade is supposed to be exposed to full south; the windows are equipped with glazing usually located inside of the facade.

The study period is the typical day consists corresponding to the hottest month (April). Table 10 shows the data obtained for the thermal characterization. Depending on the average temperature Tm and depending on the water content $\mathrm{w}$. $\lambda$ The soil used is that of Djaména whose thermal characteristics are derived from the study by [9] on the variation of the thermal conductivity so for an average temperature of $33.4^{\circ} \mathrm{C}$, we obtained the following values:

Thermophysical characteristics of cinderblock used in Table 11 obtained from the data of construction works used are:

\section{Description of the Study}

We consider a previously defined local which we give the geometrical characteristics in Figure 2 and Figure 3.

\subsection{Description of Two Configurations}

\section{Room}

\section{Creating internal loads}

The internal loads are the heat flow generated inside of the building by sources other than the conditioning system if present. They therefore involved in the heat balance of the volume of air inside the thermal zone studied. These expenses are mainly due to occupants, machines and lighting are considered according to Figure 4 [10].

We see in our study lighting and dealing with their charges is taken into account distributions made according to the following Table 12. 


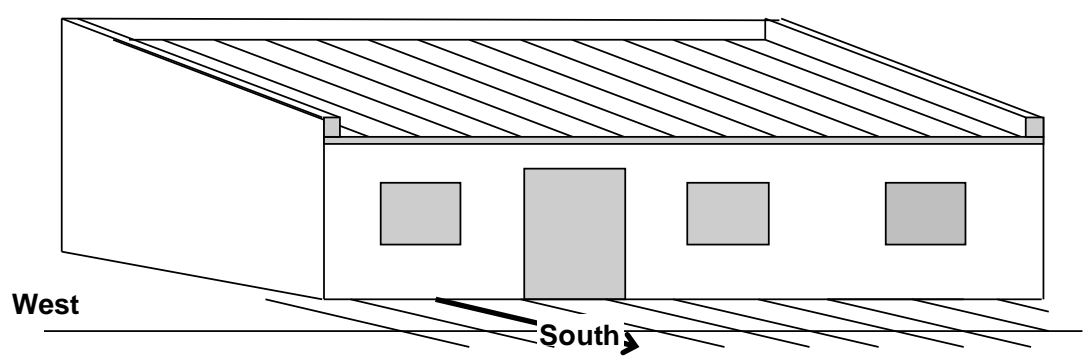

Figure 2. Overview of the study unit.

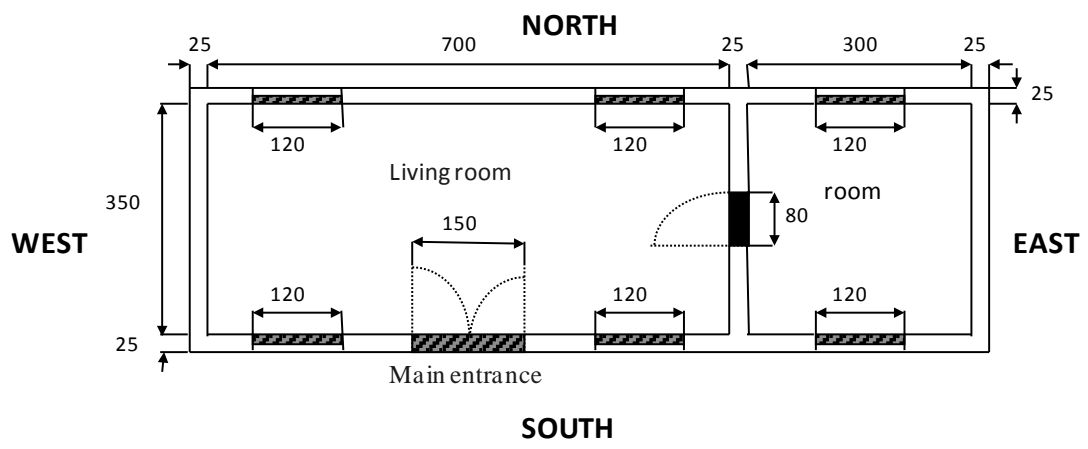

Figure 3. Plan view of the cell study.

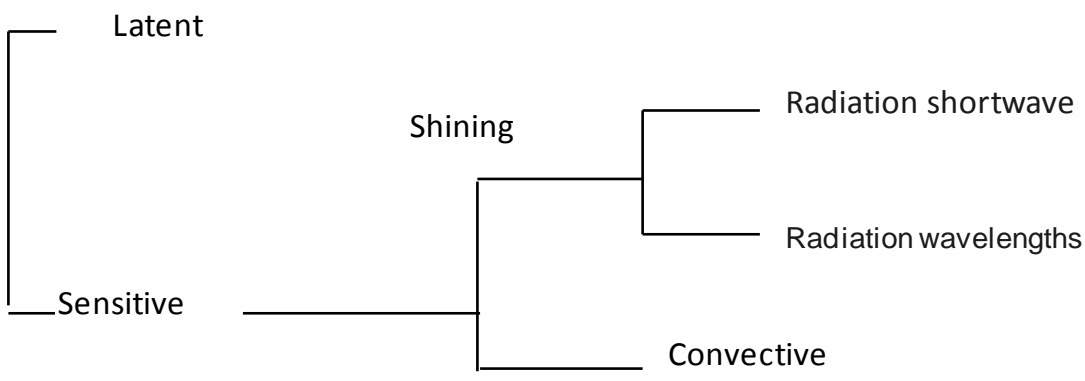

Figure 4. Schematic taking into account internal loads.

Table 10. Thermal characteristics of the land in N'Djamena.

\begin{tabular}{ccccccc}
\hline Month & $\begin{array}{c}\text { Average outdoor } \\
\text { Temperature: } T_{m}\left({ }^{\circ} \mathrm{C}\right)\end{array}$ & $\begin{array}{c}\text { Average Relative } \\
\text { Humidity Air: } H_{\text {moy }}(\%)\end{array}$ & $\begin{array}{c}\text { Average Moisture } \\
\text { content Earth: } \mathrm{w}(\%)\end{array}$ & $\begin{array}{c}\text { Apparent Thermal } \\
\text { Conductivity: } \lambda_{\text {app }}\left(\mathrm{W} / \mathrm{m} \cdot{ }^{\circ} \mathrm{C}\right)\end{array}$ & $\begin{array}{c}\text { Heat Capacity: } \\
\mathrm{C}(\mathrm{J} / \mathrm{kg} \cdot \mathrm{K})\end{array}$ & $\begin{array}{c}\text { Density: } \\
\left(\mathrm{kg} / \mathrm{m}^{3}\right)\end{array}$ \\
\hline April & 33.4 & 22.4 & 6.25 & 0.46 & 1465 & 1625 \\
\hline
\end{tabular}

Table 11. Thermal characteristics of concrete block.

\begin{tabular}{cccc}
\hline Properties & Thermal Resistance: $\mathrm{R}_{\text {th }}\left(\mathrm{m}^{2} \cdot{ }^{\circ} \mathrm{C} / \mathrm{W}\right)$ & Heat Capacity: $\mathrm{C}(\mathrm{J} / \mathrm{kg} \cdot \mathrm{K})$ & Density: $\rho\left(\mathrm{kg} / \mathrm{m}^{3}\right)$ \\
\hline Value & 0.160 & 920 & 1400 \\
\hline
\end{tabular}

Table 12. Internal charges.

\begin{tabular}{ccccccc}
\hline Load type & Number & W/unit & & Sensible & Latent \\
\hline & & & \% CLO & \% GLO & \% CONV & W/unit \\
\hline Lighting & 1 & 60 & 20 & 40 & 50 & 0 \\
Occupying & 1 & 110 & 0 & 50 & 50 \\
\hline
\end{tabular}


These internal loads are used depending on time profiles given in Table 13.

\section{Creating BATTER1 building (Earth Room)}

For Tables 14-20, the constitution of the walls of buildings using the dimensions considered and the values of thermophysical characteristics of the different measured materials or some standard values for use by the simulation with the software "CoDyBa" retained.

Table 13. Profiles of internal loads schedules.

\begin{tabular}{|c|c|c|c|c|c|c|c|c|c|c|c|c|c|c|c|}
\hline \multicolumn{8}{|c|}{ Eclairage Fluo. } & \multicolumn{8}{|c|}{ Occupant } \\
\hline \multicolumn{4}{|c|}{ Profile sensible } & \multicolumn{4}{|c|}{ Profile latent (inoperative) } & \multicolumn{4}{|c|}{ Profile sensible } & \multicolumn{4}{|c|}{ Profile latent } \\
\hline Time & $\%$ & Time & $\%$ & Time & $\%$ & Time & $\%$ & Time & $\%$ & Time & $\%$ & Time & $\%$ & Time & $\%$ \\
\hline 1 & 0 & 13 & 0 & 1 & 100 & 13 & 100 & 1 & 100 & 13 & 0 & 1 & 100 & 13 & 0 \\
\hline 2 & 0 & 14 & 0 & 2 & 100 & 14 & 100 & 2 & 100 & 14 & 0 & 2 & 100 & 14 & 0 \\
\hline 3 & 0 & 15 & 0 & 3 & 100 & 15 & 100 & 3 & 100 & 15 & 100 & 3 & 100 & 15 & 100 \\
\hline 4 & 0 & 16 & 0 & 4 & 100 & 16 & 100 & 4 & 100 & 16 & 100 & 4 & 100 & 16 & 100 \\
\hline 5 & 100 & 17 & 0 & 5 & 100 & 17 & 100 & 5 & 100 & 17 & 0 & 5 & 100 & 17 & 0 \\
\hline 6 & 100 & 18 & 100 & 6 & 100 & 18 & 100 & 6 & 100 & 18 & 0 & 6 & 100 & 18 & 0 \\
\hline 7 & 100 & 19 & 100 & 7 & 100 & 19 & 100 & 7 & 100 & 19 & 0 & 7 & 100 & 19 & 0 \\
\hline 8 & 0 & 20 & 100 & 8 & 100 & 20 & 100 & 8 & 0 & 20 & 0 & 8 & 0 & 20 & 0 \\
\hline 9 & 0 & 21 & 100 & 9 & 100 & 21 & 100 & 9 & 0 & 21 & 0 & 9 & 0 & 21 & 0 \\
\hline 10 & 0 & 22 & 100 & 10 & 100 & 22 & 100 & 10 & 0 & 22 & 0 & 10 & 0 & 22 & 0 \\
\hline 11 & 0 & 23 & 100 & 11 & 100 & 23 & 100 & 11 & 0 & 23 & 100 & 11 & 0 & 23 & 100 \\
\hline 12 & 0 & 24 & 100 & 12 & 100 & 24 & 100 & 12 & 0 & 24 & 100 & 12 & 0 & 24 & 100 \\
\hline \multicolumn{4}{|c|}{ Maximum: 60 Watt } & \multicolumn{4}{|c|}{ Maximum: 0 Watt } & \multicolumn{4}{|c|}{ Maximum: 110 Watt } & \multicolumn{4}{|c|}{ Maximum: 60 Watt } \\
\hline
\end{tabular}

Table 14. Characteristics of the south wall.

\begin{tabular}{cccccc}
\hline Layer & Material & Thickness $(\mathrm{m})$ & Conductivity: $\lambda\left(\mathrm{W} / \mathrm{m} /{ }^{\circ} \mathrm{C}.\right)$ & Cap.Cal: $\mathrm{C}\left(\mathrm{J} / \mathrm{kg} \cdot{ }^{\circ} \mathrm{C}\right)$ & Density: $\rho\left(\mathrm{kg} / \mathrm{m}^{3}\right)$ \\
\hline 1 & Coated ext. ground & 0.020 & 0.46 & 1465 & 1625 \\
2 & Bricks (ground) & 0.220 & 0.46 & 1465 & 1625 \\
3 & Int coating. ground & 0.010 & 0.46 & 1465 & 1625 \\
\hline
\end{tabular}

Table 15. Characteristics of the east wall.

\begin{tabular}{cccccc}
\hline Layer & Material & Thickness $(\mathrm{m})$ & Conductivity: $\lambda\left(\mathrm{W} / \mathrm{m} /{ }^{\circ} \mathrm{C}.\right)$ & Cap.Cal: C $\left(\mathrm{J} / \mathrm{kg} \cdot{ }^{\circ} \mathrm{C}\right)$ & Density: $\rho\left(\mathrm{kg} / \mathrm{m}^{3}\right)$ \\
\hline 1 & Coated ext. ground & 0.020 & 0.46 & 1465 & 1625 \\
2 & Bricks (ground) & 0.220 & 0.46 & 1465 & 1625 \\
3 & Int coating. ground & 0.010 & 0.46 & 1465 & 1625 \\
\hline
\end{tabular}

Table 16. Characteristics of the north wall.

\begin{tabular}{cccccc}
\hline Layer & Material & Thickness $(\mathrm{m})$ & Conductivity: $\lambda\left(\mathrm{W} / \mathrm{m} /{ }^{\circ} \mathrm{C}.\right)$ & Cap.Cal: C $\left(\mathrm{J} / \mathrm{kg} \cdot{ }^{\circ} \mathrm{C}\right)$ & Density: $\rho\left(\mathrm{kg} / \mathrm{m}^{3}\right)$ \\
\hline 1 & Coated ext. ground & 0.020 & 0.46 & 1465 & 1625 \\
2 & Bricks (ground) & 0.220 & 0.46 & 1465 & 1625 \\
3 & Int coating. ground & 0.010 & 0.46 & 1465 & 1625 \\
\hline
\end{tabular}

Table 17. Characteristics of the west wall.

\begin{tabular}{|c|c|c|c|c|c|}
\hline Layer & Material & Thickness (m) & Conductivity: $\lambda\left(\mathrm{W} / \mathrm{m} /{ }^{\circ} \mathrm{C}.\right)$ & Cap.Cal: C (J/kg· $\left.{ }^{\circ} \mathrm{C}\right)$ & Density: $\rho\left(\mathrm{kg} / \mathrm{m}^{3}\right)$ \\
\hline 1 & Coated ext. ground & 0.020 & 0.46 & 1465 & 1625 \\
\hline 2 & Bricks (ground) & 0.220 & 0.46 & 1465 & 1625 \\
\hline 3 & Int coating. ground & 0.010 & 0.46 & 1465 & 1625 \\
\hline
\end{tabular}


Table 18. Characteristics of the inside door.

\begin{tabular}{cccccc}
\hline Layer & Material & Thickness $(\mathrm{m})$ & Conductivity: $\lambda\left(\mathrm{W} / \mathrm{m} /{ }^{\circ} \mathrm{C}.\right)$ & Cap.Cal: $\mathrm{C}\left(\mathrm{J} / \mathrm{kg} \cdot{ }^{\circ} \mathrm{C}\right)$ & Density: $\rho\left(\mathrm{kg} / \mathrm{m}^{3}\right)$ \\
\hline 1 & Wood & 0.040 & 0.160 & 2095 & 800 \\
\hline
\end{tabular}

Table 19. Characteristics of the floor.

\begin{tabular}{cccccc}
\hline Layer & Material & Thickness $(\mathrm{m})$ & Conductivity: $\lambda\left(\mathrm{W} / \mathrm{m} /{ }^{\circ} \mathrm{C}.\right)$ & Cap.Cal: $\mathrm{C}\left(\mathrm{J} / \mathrm{kg} \cdot{ }^{\circ} \mathrm{C}\right)$ & $\mathrm{Density:} \rho\left(\mathrm{kg} / \mathrm{m}^{3}\right)$ \\
\hline 1 & Cement & 0.100 & 1.750 & 653 & 2100 \\
\hline
\end{tabular}

Table 20. Characteristics of roof.

\begin{tabular}{cccccc}
\hline Layer & Material & $\begin{array}{c}\text { Thermal Resistance } \\
\left(\mathrm{m}^{2} \mathrm{~K} / \mathrm{W}\right)\end{array}$ & Thickness $(\mathrm{m})$ & $\begin{array}{c}\text { Conductivity: } \lambda \\
\left(\mathrm{W} / \mathrm{m} /{ }^{\circ} \mathrm{C} .\right)\end{array}$ & $\begin{array}{c}\text { Cap.Cal: C } \\
\left(\mathrm{J} / \mathrm{kg} \cdot{ }^{\circ} \mathrm{C}\right)\end{array}$ \\
\hline 1 & Sheet metal & - & 0.002 & 70 & 800 \\
2 & Airspace & 0.180 & 0.400 & - & 100 \\
3 & Wood & - & 0.005 & 0.100 & 1000 \\
\hline
\end{tabular}

\section{a) Walls \\ 1) South Wall}

Type: outer wall

Azimuth: $0^{\circ} \quad$ Area: $6.6 \mathrm{~m}^{2} \quad$ Tilt: $90^{\circ}$

Absorption coefficient (int/ext): 0.35/0.30 Thermal Resistance: $0.554^{\circ} \mathrm{C} \cdot \mathrm{m}^{2} / \mathrm{W}$

Temperature reduction coefficient TAU: 1.00 .

\section{2) East Wall}

Type: outer wall

Azimuth: $0^{\circ} \quad$ Area: $10.5 \mathrm{~m}^{2} \quad$ Tilt: $90^{\circ}$

Absorption coefficient (int/ext): 0.35/0.30 Thermal Resistance: $0.554^{\circ} \mathrm{C} \cdot \mathrm{m}^{2} / \mathrm{W}$

Temperature reduction coefficient TAU: 1.00 .

\section{3) North Wall}

Type: outer wall

Azimuth: $0^{\circ} \quad$ Area: $9 \mathrm{~m}^{2} \quad$ Tilt: $90^{\circ}$

Absorption coefficient (int/ext): 0.35/0.30 Thermal Resistance: $0.554^{\circ} \mathrm{C} \cdot \mathrm{m}^{2} / \mathrm{W}$

Temperature reduction coefficient TAU: 1.00 .

\section{4) West Wall}

Type: outer wall

Azimuth: $0^{\circ} \quad$ Area: $8.82 \mathrm{~m}^{2} \quad$ Tilt: $90^{\circ}$

Absorption coefficient (int/ext): 0.35/0.30 Thermal Resistance: $0.554^{\circ} \mathrm{C} \cdot \mathrm{m}^{2} / \mathrm{W}$

Temperature reduction coefficient TAU: 1.00 .

\section{5) West wall 2 (door)}

Type: inside wall
Azimuth: $0^{\circ}$
Area: $1.68 \mathrm{~m}^{2}$
Tilt: $90^{\circ}$

Absorption coefficient (int/ext): 0.35/0.35 Thermal Resistance: $0.250^{\circ} \mathrm{C} \cdot \mathrm{m}^{2} / \mathrm{W}$ Temperature reduction coefficient TAU: 0.00 .

\section{6) Low floor}

Type: floor

Azimuth: $0^{\circ}$

Area: $10.50 \mathrm{~m}^{2} \quad$ Tilt: $180^{\circ}$

Absorption coefficient (int/ext): 0.50/0.00 Thermal Resistance: $0.057^{\circ} \mathrm{C} \cdot \mathrm{m}^{2} / \mathrm{W}$

Temperature reduction coefficient TAU: 0.00 .

7) Roof

Type: floor

Azimuth: $0^{\circ} \quad$ Area: $10.50 \mathrm{~m}^{2} \quad$ Tilt: $0^{\circ}$

Absorption coefficient (int/ext): 0.35/0.50 Thermal Resistance: $0.759^{\circ} \mathrm{C} \cdot \mathrm{m}^{2} / \mathrm{W}$

Temperature reduction coefficient TAU: 1.00 . 


\section{b) Windows}

1) North Window

Number of windows: 1

Height: $1.20 \mathrm{~m}$

Width: $1 \mathrm{~m}$

Percentage of opening: $50 \%$

Azimuth: $180^{\circ}$

Tilt: $90^{\circ}$

Depth: $0.25 \mathrm{~m}$

K Day/Night: 4.8

Permeability: 4

Clear coefficient: 0.70

Window reads: glazed windows

Number of single: 1

Single Glass: transmission coefficient (0.85); absorption coefficient (0.08).

\section{2) South Window}

Number of windows: 1

Height: $1.20 \mathrm{~m}$

Azimuth: $0^{\circ}$

Width: $1 \mathrm{~m}$

Percentage of opening: $50 \%$

K Day/Night: 4.8

Tilt: $90^{\circ}$

Depth: $0.25 \mathrm{~m}$

Window reads: glazed windows

Permeability: 4

Clear coefficient: 0.70

Number of single: 1

Single Glass: transmission coefficient (0.85); absorption coefficient $(0.08)$.

\section{Creating BATBET1 building (Cement Room)}

The data cited in the beginning of each paragraph (a1-a7) to determine the characteristics of the southern walls, East, North, West, and the inner door, the floor and roof in Tables 21-27.

Table 21. Characteristics of the south wall (cement).

\begin{tabular}{ccccccc}
\hline Layer & Material & $\begin{array}{c}\text { Thermal Resistance } \\
\left(\mathrm{m}^{2} \mathrm{~K} / \mathrm{W}\right)\end{array}$ & Thickness $(\mathrm{m})$ & $\begin{array}{c}\text { Conductivity: } \lambda \\
\left(\mathrm{W} / \mathrm{m} /{ }^{\circ} \mathrm{C} .\right)\end{array}$ & $\begin{array}{c}\text { Cap.Cal: C } \\
\left(\mathrm{J} / \mathrm{kg} \cdot{ }^{\circ} \mathrm{C}\right)\end{array}$ & Density: $\rho\left(\mathrm{kg} / \mathrm{m}^{3}\right)$ \\
\hline 1 & Coated ext. cement & - & 0.020 & 1.150 & 1000 & 1700 \\
2 & Blocks (cement) & 0.160 & 0.220 & - & 920 & 1400 \\
3 & Coated Int. cement & - & 0.010 & 1.150 & 1000 & 1700 \\
\hline
\end{tabular}

Table 22. Characteristics of the east wall (cement).

\begin{tabular}{ccccccc}
\hline Layer & Material & $\begin{array}{c}\text { Thermal Resistance } \\
\left(\mathrm{m}^{2} \mathrm{~K} / \mathrm{W}\right)\end{array}$ & Thickness $(\mathrm{m})$ & $\begin{array}{c}\text { Conductivity: } \lambda \\
\left(\mathrm{W} / \mathrm{m} /{ }^{\circ} \mathrm{C} .\right)\end{array}$ & $\begin{array}{c}\text { Cap.Cal: C } \\
\left(\mathrm{J} / \mathrm{kg} \cdot{ }^{\circ} \mathrm{C}\right)\end{array}$ & Density: $\rho\left(\mathrm{kg} / \mathrm{m}^{3}\right)$ \\
\hline 1 & Coated ext. cement & - & 0.020 & 1.150 & 1000 & 1700 \\
2 & Blocks (cement) & 0.160 & 0.220 & - & 920 & 1400 \\
3 & Coated Int. cement. & - & 0.010 & 1.150 & 1000 & 1700 \\
\hline
\end{tabular}

Table 23. Characteristics of the North wall (cement).

\begin{tabular}{cccccc}
\hline Layer & Material & $\begin{array}{c}\text { Thermal Resistance } \\
\left(\mathrm{m}^{2} \mathrm{~K} / \mathrm{W}\right)\end{array}$ & Thickness $(\mathrm{m})$ & $\begin{array}{c}\text { Conductivity: } \lambda \\
\left(\mathrm{W} / \mathrm{m} /{ }^{\circ} \mathrm{C} .\right)\end{array}$ & $\begin{array}{c}\text { Cap.Cal: } \mathrm{C} \\
\left(\mathrm{J} / \mathrm{kg} \cdot{ }^{\circ} \mathrm{C}\right)\end{array}$ \\
\hline 1 & Coated ext. cement & - & 0.020 & 1.150 & 1000 \\
2 & Blocks (cement) & 0.160 & 0.220 & - & 920 \\
3 & Coated Int. cement & - & 0.010 & 1.150 & 1700 \\
\hline
\end{tabular}

Table 24. Characteristics of the west wall (cement).

\begin{tabular}{ccccccc}
\hline Layer & Material & $\begin{array}{c}\text { Thermal Resistance } \\
\left(\mathrm{m}^{2} \mathrm{~K} / \mathrm{W}\right)\end{array}$ & Thickness $(\mathrm{m})$ & $\begin{array}{c}\text { Conductivity: } \lambda \\
\left(\mathrm{W} / \mathrm{m} /{ }^{\circ} \mathrm{C} .\right)\end{array}$ & $\begin{array}{c}\text { Cap.Cal: C } \\
\left(\mathrm{J} / \mathrm{kg} \cdot{ }^{\circ} \mathrm{C}\right)\end{array}$ & Density: $\rho\left(\mathrm{kg} / \mathrm{m}^{3}\right)$ \\
\hline 1 & Coated ext. cement & - & 0.015 & 1.150 & 1000 & 1700 \\
2 & Blocks (cement) & 0.160 & 0.220 & - & 920 & 1400 \\
3 & Coated Int. cement & - & 0.015 & 1.150 & 1000 & 1700 \\
\hline
\end{tabular}

Table 25. Characteristics of the inside door.

\begin{tabular}{cccccc}
\hline Layer & Material & Thickness $(\mathrm{m})$ & $\begin{array}{c}\text { Conductivity: } \lambda \\
\left(\mathrm{W} / \mathrm{m} /{ }^{\circ} \mathrm{C} .\right)\end{array}$ & $\begin{array}{c}\text { Cap.Cal: } \mathrm{C} \\
\left(\mathrm{J} / \mathrm{kg} \cdot{ }^{\circ} \mathrm{C}\right)\end{array}$ & Density: $\rho\left(\mathrm{kg} / \mathrm{m}^{3}\right)$ \\
\hline 1. & Wood & 0.040 & 0.160 & 2095 & 800 \\
\hline
\end{tabular}


Table 26. Characteristics of the floor.

\begin{tabular}{|c|c|c|c|c|c|c|}
\hline Layer & Material & Thickness (m) & Conductivity: $\lambda(\mathrm{W} / \mathrm{m} /$ & Cap.Ca & $\left(\mathrm{J} / \mathrm{kg} \cdot{ }^{\circ} \mathrm{C}\right)$ & Density: $\rho\left(\mathrm{kg} / \mathrm{m}^{3}\right)$ \\
\hline 1 & cement & 0.100 & 1.750 & \multicolumn{2}{|c|}{653} & 2100 \\
\hline Layer & Material & $\begin{array}{l}\text { Thermal Resistance } \\
\left(\mathrm{m}^{2} \mathrm{~K} / \mathrm{W}\right)\end{array}$ & Thickness (m) & $\begin{array}{l}\text { Conductivity: } \lambda \\
\left(\mathrm{W} / \mathrm{m} /{ }^{\circ} \mathrm{C} .\right)\end{array}$ & $\begin{array}{l}\text { Cap.Cal: C } \\
\left(\mathrm{J} / \mathrm{kg} \cdot{ }^{\circ} \mathrm{C}\right)\end{array}$ & $\begin{array}{l}\text { Density: } \rho \\
\left(\mathrm{kg} / \mathrm{m}^{3}\right)\end{array}$ \\
\hline 1. & Sheet metal & - & 0.002 & 70 & 800 & 7000 \\
\hline 2 & Airspace & 0.180 & 0.400 & - & 100 & 1000 \\
\hline 3 & Wood & - & 0.005 & 0.100 & 990 & 1200 \\
\hline
\end{tabular}

\section{a) Walls \\ 1) South Wall}

Type: outer wall
Azimuth: $0^{\circ}$
Area: $6.6 \mathrm{~m}^{2}$
Tilt: $90^{\circ}$

Absorption coefficient (int/ext): 0.35/0.30 Thermal Resistance: $0.071^{\circ} \mathrm{C} \cdot \mathrm{m}^{2} / \mathrm{W}$

Temperature reduction coefficient TAU: 1.00 .

\section{2) East Wall}

Type: outer wall

Azimuth: $-90^{\circ} \quad$ Area: $10.5 \mathrm{~m}^{2} \quad$ Tilt: $90^{\circ}$

Absorption coefficient (int/ext): 0.35/0.30 Thermal Resistance: $0.071^{\circ} \mathrm{C} \cdot \mathrm{m}^{2} / \mathrm{W}$

Temperature reduction coefficient TAU: 1.00 .

\section{3) North Wall}

Type: outer wall

Azimuth: $180^{\circ} \quad$ Area: $9 \mathrm{~m}^{2} \quad$ Tilt: $90^{\circ}$

Absorption coefficient (int/ext): 0.35/0.30 Thermal Resistance: $0.071^{\circ} \mathrm{C} \cdot \mathrm{m}^{2} / \mathrm{W}$

Temperature reduction coefficient TAU: 1.00 .

\section{4) West Wall}

Type: Inside wall
Azimuth: $90^{\circ}$
Area: $8.82 \mathrm{~m}^{2}$
Tilt: $90^{\circ}$

Absorption coefficient (int/ext): 0.35/0.30 Thermal Resistance: $0.067^{\circ} \mathrm{C} \cdot \mathrm{m}^{2} / \mathrm{W}$ Temperature reduction coefficient TAU: 0.00 .

\section{5) West Wall 2 (door)}

Type: inside wall
Azimuth: $0^{\circ}$
Area: $1.68 \mathrm{~m}^{2}$
Tilt: $90^{\circ}$

Absorption coefficient (int/ext): 0.35/0.35 Thermal Resistance: $0.250^{\circ} \mathrm{C} \cdot \mathrm{m}^{2} / \mathrm{W}$

Temperature reduction coefficient TAU: 0.00 .

6) Low Floor

Type: floor

Azimuth: $0^{\circ} \quad$ Area: $10.50 \mathrm{~m}^{2} \quad$ Tilt: $180^{\circ}$

Absorption coefficient (int/ext): 0.50/0.00 Thermal Resistance: $0.057^{\circ} \mathrm{C} \cdot \mathrm{m}^{2} / \mathrm{W}$

Temperature reduction coefficient TAU: 0.00 .

\section{7) Roof}

Type: floor

Azimuth: $0^{\circ} \quad$ Area: $10.50 \mathrm{~m}^{2} \quad$ Tilt: $0^{\circ}$

Absorption coefficient (int/ext): 0.35/0.50 Thermal Resistance: $0.759^{\circ} \mathrm{C} \cdot \mathrm{m}^{2} / \mathrm{W}$

Temperature reduction coefficient TAU: 1.00 .

\section{b) Windows}

\section{1) North Window}

Number of windows: 1

Height: $1.20 \mathrm{~m}$

Width: $1 \mathrm{~m}$

Percentage of opening: $50 \%$

Depth: $0.25 \mathrm{~m}$ 
Azimuth: $180^{\circ}$

K Day/Night: 4.8

Window reads: glazed windows
Permeability: 4

Clear coefficient: 0.70

Number of single: 1

Single Glass: transmission coefficient (0.85); absorption coefficient $(0.08)$.

\section{2) South Window}

Number of windows: 1

Height: $1.20 \mathrm{~m}$

Azimuth: $0^{\circ}$

K Day/Night: 4.8

Window reads: glazed windows

Width: $1 \mathrm{~m}$

Tilt: $90^{\circ}$

Single Glass: transmission coefficient (0.85); absorption coefficient (0.08).

\subsection{Simulation Results}

Simulation results give us as defined configurations of the selected habitat changes per hour during 24 hours of a hot day in the hottest months, the following quantities:

- The temperature of the ambient air within the space;

- The resulting temperature of the atmosphere;

- And the local relative humidity.

We present in following Table 28, the results of all local defined in tables and variations of these quantities with time.

Table 28. Results of thermal simulation rooms.

\begin{tabular}{|c|c|c|c|c|c|c|}
\hline \multirow{2}{*}{ Time } & \multicolumn{3}{|c|}{ Room : Batter1 } & \multicolumn{3}{|c|}{ Cement room: Batbet1 } \\
\hline & $\mathrm{T}^{\circ}$ air int. $\left({ }^{\circ} \mathrm{C}\right)$ & Tres. $\left({ }^{\circ} \mathrm{C}\right)$ & $\mathrm{Hr}(\%)$ & $\mathrm{T}^{\circ}$ air int. $\left({ }^{\circ} \mathrm{C}\right)$ & Tres $\left({ }^{\circ} \mathrm{C}\right)$ & $\mathrm{Hr}(\%)$ \\
\hline 1 & 31.6 & 32.6 & 28 & 32.1 & 33.2 & 27 \\
\hline 2 & 31.2 & 32.2 & 29 & 31.4 & 32.5 & 29 \\
\hline 3 & 30.9 & 31.9 & 29 & 30.9 & 32.0 & 29 \\
\hline 4 & 30.8 & 31.7 & 30 & 30.6 & 31.5 & 30 \\
\hline 5 & 31.0 & 31.7 & 29 & 30.6 & 31.4 & 30 \\
\hline 6 & 31.3 & 31.8 & 29 & 30.8 & 31.3 & 30 \\
\hline 7 & 32.9 & 32.6 & 35 & 32.1 & 31.9 & 37 \\
\hline 8 & 32.7 & 32.5 & 36 & 31.9 & 31.7 & 37 \\
\hline 9 & 32.8 & 32.6 & 36 & 32.1 & 32.0 & 37 \\
\hline 10 & 32.9 & 32.9 & 35 & 32.5 & 32.4 & 36 \\
\hline 11 & 33.1 & 33.1 & 35 & 32.9 & 32.9 & 35 \\
\hline 12 & 33.3 & 33.4 & 35 & 33.4 & 33.4 & 35 \\
\hline 13 & 33.6 & 33.6 & 34 & 33.9 & 33.9 & 34 \\
\hline 14 & 33.8 & 33.9 & 34 & 34.4 & 34.5 & 33 \\
\hline 15 & 34.3 & 34.4 & 41 & 35.2 & 35.2 & 39 \\
\hline 16 & 34.5 & 34.6 & 49 & 35.7 & 35.7 & 46 \\
\hline 17 & 35.2 & 35.0 & 27 & 36.5 & 36.2 & 25 \\
\hline 18 & 35.3 & 35.1 & 23 & 36.6 & 36.5 & 21 \\
\hline 19 & 35.5 & 35.3 & 22 & 36.7 & 36.6 & 21 \\
\hline 20 & 35.0 & 35.0 & 23 & 36.1 & 36.3 & 21 \\
\hline 21 & 34.3 & 34.6 & 23 & 35.4 & 35.8 & 22 \\
\hline 22 & 33.6 & 34.1 & 24 & 34.5 & 35.2 & 23 \\
\hline 23 & 33.0 & 33.8 & 26 & 33.8 & 34.7 & 25 \\
\hline 24 & 32.3 & 33.2 & 27 & 33.0 & 34.0 & 26 \\
\hline
\end{tabular}




\section{* Room}

In Figures 5-8, we have shown the resulting curves of the simulation of the two cases studied configurations, in order to observe the variation of the temperature of indoor air, the relative humidity and the resulting temperature for 24 hours.

From these curves, one notes that the amplitude of variations of these amenities (the temperature of indoor air, the relative humidity and the resulting temperature) is slightly lower for the earth construction as that of the construction cement. Indeed, during the hottest hours of the day, the thermal inertia of earth constructions tends to maintain the temperature of the indoor local air above the cement constructions.

We can say that for a hot month in Chad, it is more comfortable to live in a thermally land habitat because generally a local air temperature, relative humidity and high mean radiant temperature tend to produce a sensation discomfort in quiet air.

\section{Thermal Comfort}

Thermal comfort is considered the expressed satisfaction or subjective indifference to the local atmosphere. It is, indeed, based on heat exchange between the human body and environment. The comfort is even better than the heat generated by the body (metabolism) can be disengaged with less stress.

To define thermal comfort conditions, diagrams were long used moist air which are bounded on comfort zones (e.g. Givoni diagram: Annex A4). Using these diagrams sketchy faces a problem of choice: chart contour zones vary according to the authors.

To determine the thermal sensation of an individual characteristic data in a given environment, we will use a more efficient method is the model of Fanger [9] [11].

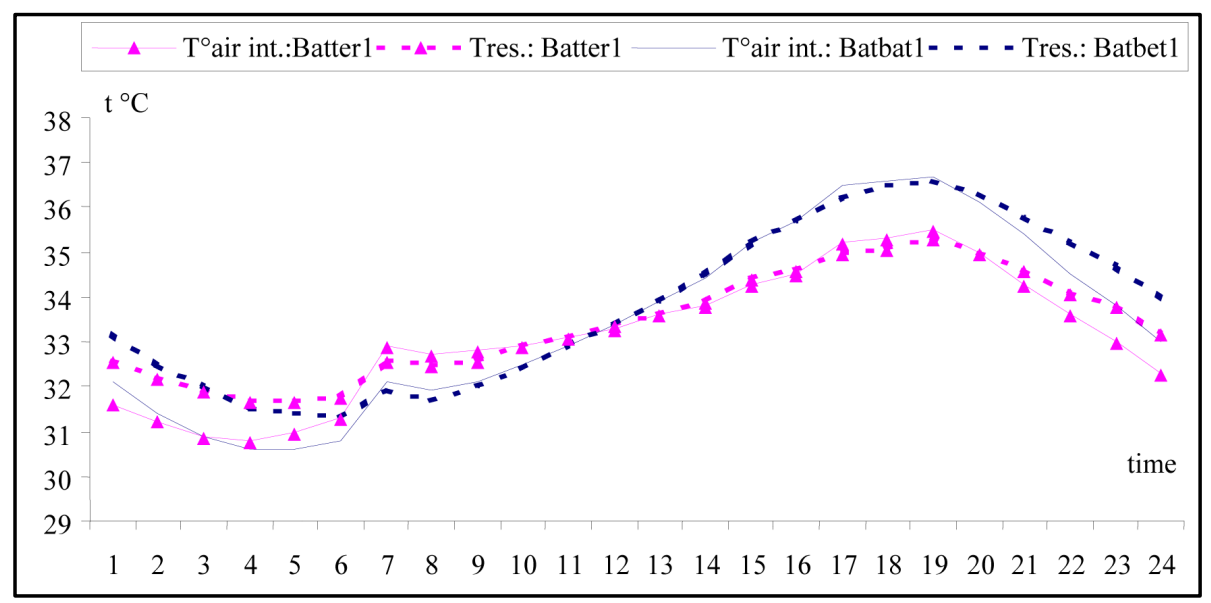

Figure 5. Variation of the resulting temperature and the air temperature inside the rooms.

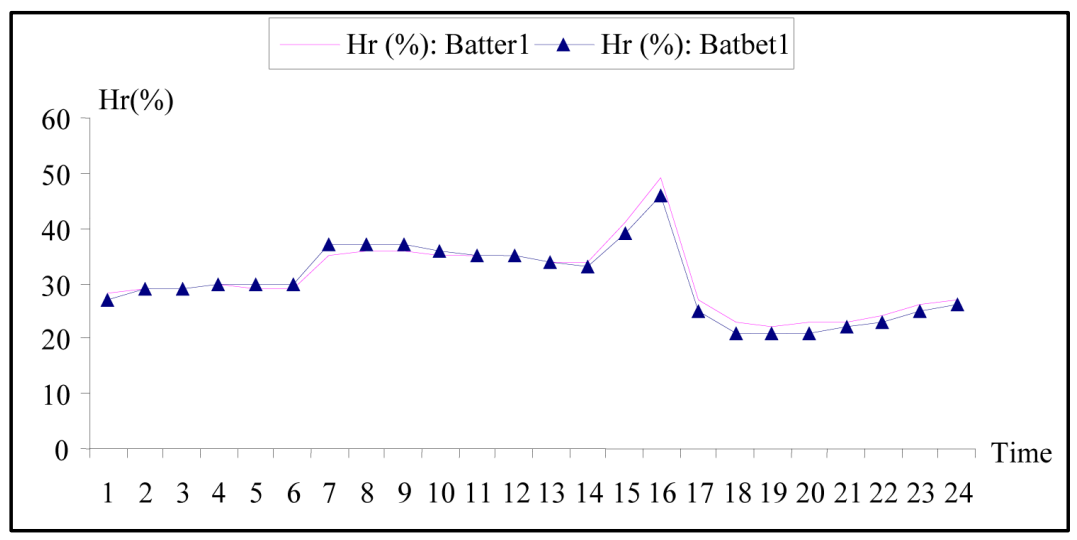

Figure 6. Variation of relative humidity rooms. 


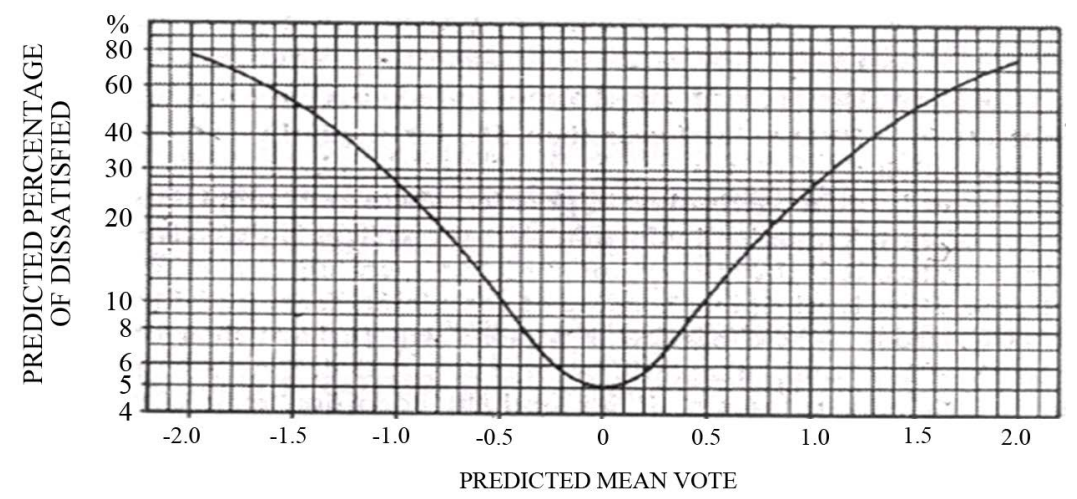

Figure 7. PPD evolution according to PMV.

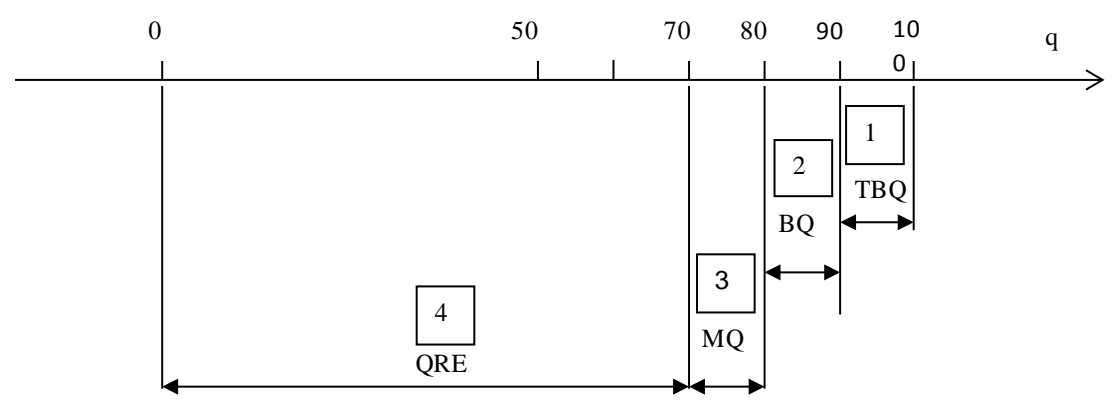
1.: TBQ: high quality habitat
2.: BQ: good quality habitat
3. MQ: medium quality habitat
4.: QRE: poor quality habitat

Figure 8. habitat quality characterization Scale.

\subsection{The Model of Fanger}

The equation of the instantaneous humidity heat balance between the human body and its environment is written [12]-[14]:

$$
\begin{gathered}
E_{r y}=3.95 \times 10^{-8} F_{c l} \cdot A_{d u}\left[\left(T_{c l}+273\right)^{4}-\left(T_{r}+273\right)^{4}\right] \\
E_{c v}=F_{c l} \cdot h_{c}\left(T_{c l}-T_{a}\right) \\
E_{p s}=(M-W)-3.05 \times 10^{-3}\left[5733-6.99(M-W)-P_{v}\right] \\
E_{c r}=0.00146 M\left(34-T_{a}\right) \\
E_{h r}=1.73 \times 10^{-5} M\left(5820-P_{v}\right) \\
E_{s d}=0.42[(M-W)-58.15]
\end{gathered}
$$

avec:

$F_{c l}$ : ratio between the area covered by the coat and the surface of the naked body

$$
\begin{gathered}
* F_{c l}=1+0.2 I_{c l} \quad s i I_{c l}=0.5 \text { Clo } \\
* F_{c l}=1.05+0.1 I_{c l} \quad s i I_{c l}>0.5 \mathrm{Clo}
\end{gathered}
$$

$h c$ : exchange coefficient between the skin and clothing given by: 


$$
\begin{gathered}
* h c=2.38\left(T_{c l}-T_{a}\right)^{0.25} \quad \text { si } 2.38\left(T_{c l}-T_{a}\right)>12.1 \sqrt{v} \\
* h c=12.1 \sqrt{v} \quad \text { si } 2.38\left(T_{c l}-T_{a}\right)<12.1 \sqrt{v}
\end{gathered}
$$

$T_{r}$ : mean radiant temperature;

$T_{a}$ : temperature of the ambient air;

$P_{v}$ : partial pressure of water vapor at the temperature of the ambient air;

$T_{c l}$ : Living the temperature determined by:

$$
{ }^{*} T_{c l}=T_{c u t}-0.155 I_{c l}\left(M-W-E_{p s}-E_{r h}-E_{c r}-E_{s d}\right)
$$

$T_{\text {cut }}$ skin temperature calculated by

$$
* T_{\text {cut }}=29.35+0.196 T_{a}-1.064 \frac{M}{58.15}
$$

By replacing the terms of the equation:

$$
Q=(M-W)-\left(E_{r y}+E_{c v}+E_{c d}+E_{p s}+E_{c r}+E_{h r}+E_{s d}\right)
$$

by their value while laying $H=M-W$ and dividing by $A_{d u}$ we obtain the thermal equation of the individual who is:

$$
\begin{aligned}
\frac{Q}{A_{d u}}= & \frac{H}{A_{d u}}-\frac{1}{A_{d u}}\left\{(M-W)-3.05 \times 10^{-3}\left[5733-6.99(M-W)-P_{v}\right]\right\} \\
& -0.00146 \frac{M}{A_{d u}}\left(34-T_{a}\right)-1.73 \times 10^{-5} \frac{M}{A_{d u}}\left(5820-P_{v}\right)-\frac{0.42}{A_{d u}}[(M-W)-58.15] \\
& -3.95 \times 10^{-8} F_{c l} \cdot\left[\left(T_{c l}+273\right)^{4}-\left(T_{r}+273\right)^{4}\right]-F_{c l} \cdot h_{c}\left(T_{c l}-T_{a}\right) .
\end{aligned}
$$

Fanger proposed formulas for calculating each of the speaker terms in equation (10) the thermal balance of the body in a state of thermal comfort. These formulas were derived from experimental studies involving a large number of subjects (1300) and include both physical and physiological measurements and the record subjective assessments of the test subjects.

The formulation of Fanger is recognized worldwide today and has replaced the use of comfort zones in the humid air diagram.

Fanger determines the vicinity of the comfort zone a number of thermal sensation levels experienced by an individual. In a situation of comfort, thermal equilibrium is achieved without recourse to sweat: that is to say, the thermal load of the individual is zero. The feeling of comfort is translated by the following equation:

$$
Q=(M-W)-\left(E_{r y}+E_{c v}+E_{p s}+E_{c r}+E_{h r}+E_{s}\right)=0
$$

The deviation from the ideal conditions of comfort is characterized by the PMV index (Predicted Mean Vote). This index to quantify the thermal sensation, represents the average value of ratings of a sample of individuals in response to thermal stresses atmosphere.

This index is calculated from the heat balance imbalance:

$$
P M V=\frac{Q \cdot C_{a}}{A_{d u}}
$$

where $C_{a}=0.303 \cdot \mathrm{e}^{-2.1 \cdot A C T}+0.028, A C T$ : MET activity parameter $\left(1 \mathrm{MET}=58.15 \mathrm{~W} / \mathrm{m}^{2}\right)$

Each level of thermal sensation is identified numerically according the following Table 29:

The PMV is the average value of votes, Fanger has therefore sought to know the percentage of dissatisfied thermal sensation zone. It has introduced another PPD index (Predicted Percentage of Dissatisfied). This index will help in determining rationally quality ambience of a room for a certain type of activity. The PPD is connected to the VMS by Equation (13): 
Table 29. Qualification scale thermal sensation.

\begin{tabular}{cc}
\hline PMV & Thermal sensation \\
+3 & hot \\
+2 & lukewarm \\
+1 & slightly warm \\
0 & neutral \\
-1 & slightly chilled \\
-2 & fresh \\
-3 & cold \\
$P P D=100-95 \exp \left[-\left(0.03353 P M V^{4}+0.2179 P M V^{2}\right)\right]$
\end{tabular}

We can then find the values of PPD from the curve of Figure 7.

The function score comfort $\mathrm{n}$ is built from the PPD by Equation (14):

$$
n=100-P P D
$$

Either:

$$
n=95 \exp \left[-\left(0.03353 P M V^{4}+0.2179 P M V^{2}\right)\right]
$$

$q$ that would account number of the thermal quality of habitat is based on ratings comfort $n$ (t) corresponding to the times $t$ studied. As part of the contribution to the development of the general laws of design assistance, taking into account the average daily need more locally because they illustrate the evolution of the quality score changes induced by the project designer. These qj daily notes, which are expressed by equation (15):

$$
q_{j}=\frac{\left[\sum_{i=1}^{i=24} n_{i, j}\right]}{24}
$$

sometimes will isolate a particularly typical day and observe the changes in results of the analysis comfortable conditions on a specific subsequence, thus we consider a typical day's hottest April to review by the characterization given by scale [14] and shown in Figure 8 the two cases selected configuration.

\subsection{Application of the Model of Fanger}

We consider a standard user sitting at rest in the various local defined above, in April, with a light summer dress. We will evaluate the balance of heat exchanges that individual and its environment to determine the feeling of thermal comfort in each local defined above. This sensation can be evaluated from the resulting temperature very dry, which summarizes the radiative and convective exchanges. This temperature is given by the following expression:

$$
T_{\text {res }}=\frac{h_{c i} T_{\text {air.int }}+h_{r} T_{r}}{h_{c i}+h_{r}}
$$

with: $h_{c i}$, exchange coefficient by convection;

$h_{r}$, radiant exchange coefficient;

$T_{r}$, mean radiant temperature;

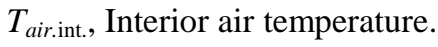

In still air and the $h_{c i}$ and $h_{r}$ coefficients are very close to where we have: $T_{\text {res }}=\frac{T_{\text {air.int. }}+T_{r}}{2}$

The values of dry resulting temperature we are given by the simulation results.

\subsubsection{Characteristics Ambience}

The characteristics of the local atmosphere are determined for a day. The values of the temperature of the interior air, the resulting temperature and relative humidity are obtained by simulation. Those of mean radiant tem- 
perature and vapor pressure are derived from the values of the quantities mentioned above. Table 30 shows the values of the characteristics of the atmosphere of rooms.

\subsubsection{Individual Characteristics and Dress}

For standard individual of $1.73 \mathrm{~m}$ height and $70 \mathrm{~kg}$ weight sitting at rest and wearing a light summer dress, the thermal characteristics of the person and his vesture are summarized in the following Table 31.

\subsubsection{Evaluation of the Thermal Quality}

In this section, we will evaluate the different terms of thermo hygrometric equation, given by equations (1)-(6). From these equations and results of various characteristics of the elements identified above, we will calculate the

Table 30. Characteristics of the ambiance of Room.

\begin{tabular}{|c|c|c|c|c|c|c|c|c|}
\hline \multirow{2}{*}{ Time } & \multicolumn{4}{|c|}{ Ground room } & \multicolumn{4}{|c|}{ Cement room } \\
\hline & $T_{\text {air.int. }}\left({ }^{\circ} \mathrm{C}\right)$ & $T_{r}\left({ }^{\circ} \mathrm{C}\right)$ & $H_{r \text { int. }}(\%)$ & $\mathrm{Pv}(\mathrm{mb})$ & $T_{\text {air.int. }}\left({ }^{\circ} \mathrm{C}\right)$ & $T_{r}\left({ }^{\circ} \mathrm{C}\right)$ & $H_{r \text { int. }}(\%)$ & $\mathrm{Pv}(\mathrm{mb})$ \\
\hline 1 & 31.6 & 33.2 & 28 & 13.53 & 32.1 & 33.9 & 27 & 13.40 \\
\hline 2 & 31.2 & 32.7 & 29 & 13.71 & 31.4 & 33.0 & 29 & 13.6 \\
\hline 3 & 30.9 & 32.3 & 29 & 13.48 & 30.9 & 32.2 & 29 & 13.48 \\
\hline 4 & 30.8 & 31.8 & 30 & 13.87 & 30.6 & 31.5 & 30 & 13.72 \\
\hline 5 & 31.0 & 31.5 & 29 & 13.56 & 30.6 & 30.9 & 30 & 13.72 \\
\hline 6 & 31.3 & 31.3 & 29 & 13.78 & 30.8 & 30.7 & 30 & 13.87 \\
\hline 7 & 32.9 & 31.3 & 35 & 18.15 & 32.1 & 30.5 & 37 & 18.37 \\
\hline 8 & 32.7 & 31.4 & 36 & 18.46 & 31.9 & 30.7 & 37 & 18.17 \\
\hline 9 & 32.8 & 31.7 & 36 & 18.57 & 32.1 & 31.0 & 37 & 18.37 \\
\hline 10 & 32.9 & 32.1 & 35 & 18.15 & 32.5 & 31.5 & 36 & 18.27 \\
\hline 11 & 33.1 & 32.5 & 35 & 18.35 & 32.9 & 32.0 & 35 & 18.15 \\
\hline 12 & 33.3 & 32.8 & 35 & 18.54 & 33.4 & 32.6 & 35 & 18.65 \\
\hline 13 & 33.6 & 33.4 & 34 & 18.31 & 33.9 & 33.4 & 34 & 18.61 \\
\hline 14 & 33.8 & 33.7 & 34 & 18.51 & 34.4 & 34.1 & 33 & 18.55 \\
\hline 15 & 34.3 & 34.0 & 41 & 22.93 & 35.2 & 34.7 & 39 & 22.89 \\
\hline 16 & 34.5 & 34.5 & 49 & 27.70 & 35.7 & 35.4 & 46 & 27.72 \\
\hline 17 & 35.2 & 34.6 & 27 & 15.84 & 36.5 & 35.9 & 25 & 15.72 \\
\hline 18 & 35.3 & 34.8 & 23 & 13.57 & 36.6 & 36.4 & 21 & 13.27 \\
\hline 19 & 35.5 & 35.1 & 22 & 13.12 & 36.7 & 36.7 & 21 & 13.35 \\
\hline 20 & 35.0 & 35.1 & 23 & 13.35 & 36.1 & 36.8 & 21 & 12.93 \\
\hline 21 & 34.3 & 34.9 & 23 & 12.86 & 35.4 & 36.4 & 22 & 13.05 \\
\hline 22 & 33.6 & 34.8 & 24 & 12.92 & 34.5 & 36.0 & 23 & 13.00 \\
\hline 23 & 33.0 & 34.2 & 26 & 13.55 & 33.8 & 35.3 & 25 & 13.61 \\
\hline 24 & 32.3 & 33.7 & 27 & 13.55 & 33.0 & 34.5 & 26 & 13.55 \\
\hline
\end{tabular}

Table 31. Characteristics of the individual and of his clothing.

\begin{tabular}{cc}
\hline Activity: Sitting at rest & Cladding: Wear lightweight summer \\
Metabolism $(\mathrm{M})=106 \mathrm{w}$ & Total thermal resistance $=0.078 \mathrm{~m}{ }^{\circ}{ }^{\circ} \mathrm{C} / \mathrm{W}$ \\
Mechanical power ext. $(\mathrm{W})=0 \mathrm{~W}$ & Isolation of the dress $=0.5 \mathrm{Clo}$ \\
\hline
\end{tabular}


different indices PMV and PPD comfort [12] and No scoring function given by equation (14). The results of this calculation are presented in Tables 32-34.

*Room

Given that ISO 7730 [15] provides acceptable thermal comfort for the following values for the PMV and PPD:

- $-5<\mathrm{PMV}<5$

- $\mathrm{PPD}<10 \%$

Note that the PMV and PPD indices as they have been defined by Fanger, are global indices applying to the human body and the local in its entirety (using air temperature, radiant temperature, of humidity and air velocity) per hour. We made the calculation of average daily indices of these premises to use as a simple way to characterize thermal comfort of the subject therein. Given the results of this calculation, we can say that thermal comfort is acceptable in both configurations.

From the PMV values, we used the relation (4) to determine the score of comfort $n$ because one of the advantages of this rating system is to avoid physical Manichaeism declaring comfortable or not comfortable atmosphere that according one is located or not in the thermal comfort zone. The system proposed by [16]-[18] allows

Table 32. Calculation of comfort indices PMV and PPD ground Room.

\begin{tabular}{|c|c|c|c|c|c|c|c|c|c|}
\hline \multirow{2}{*}{ Time } & \multicolumn{9}{|c|}{ Gound room } \\
\hline & $E_{r y}$ & $E_{c v}$ & $E_{p s}$ & $E_{c r}$ & $E_{h r}$ & $E_{\text {sd }}$ & $Q$ & $P M V$ & $P P D$ \\
\hline 1 & 11.66 & 13.12 & 94.95 & 0.37 & 8.16 & 20.10 & -42.35 & -0.65 & 13.91 \\
\hline 2 & 14.40 & 14.39 & 95.01 & 0.43 & 8.13 & 20.10 & -46.46 & -0.71 & 15.75 \\
\hline 3 & 16.43 & 15.34 & 94.94 & 0.48 & 8.17 & 20.10 & -49.45 & -0.76 & 17.19 \\
\hline 4 & 18.81 & 15.67 & 95.06 & 0.50 & 8.10 & 20.10 & -52.22 & -0.80 & 18.61 \\
\hline 5 & 20.76 & 15.02 & 94.96 & 0.46 & 8.15 & 20.10 & -53.46 & -0.82 & 19.27 \\
\hline 6 & 22.08 & 14.08 & 95.03 & 0.42 & 8.11 & 20.10 & -53.82 & -0.83 & 19.46 \\
\hline 7 & 24.95 & 9.17 & 96.38 & 0.17 & 7.30 & 20.10 & -52.07 & -0.80 & 18.53 \\
\hline 8 & 24.66 & 9.81 & 96.48 & 0.20 & 7.24 & 20.10 & -52.50 & -0.81 & 18.76 \\
\hline 9 & 24.00 & 9.50 & 96.51 & 0.19 & 7.22 & 20.10 & -51.51 & -0.79 & 18.24 \\
\hline 10 & 19.95 & 9.17 & 96.38 & 0.17 & 7.30 & 20.10 & -47.07 & -0.72 & 16.04 \\
\hline 11 & 18.61 & 8.54 & 96.44 & 0.14 & 7.26 & 20.10 & -45.09 & -0.69 & 15.12 \\
\hline 12 & 15.58 & 7.91 & 96.50 & 0.11 & 7.23 & 20.10 & -41.43 & -0.64 & 13.53 \\
\hline 13 & 15.19 & 6.95 & 96.43 & 0.06 & 7.27 & 20.10 & -40.01 & -0.62 & 12.95 \\
\hline 14 & 12.15 & 6.33 & 96.49 & 0.03 & 7.23 & 20.10 & -36.33 & -0.56 & 11.54 \\
\hline 15 & 9.07 & 4.91 & 97.86 & -0.05 & 6.41 & 20.10 & -32.30 & -0.50 & 10.16 \\
\hline 16 & 8.08 & 4.45 & 99.33 & -0.08 & 5.53 & 20.10 & -31.41 & -0.48 & 9.88 \\
\hline 17 & 7.35 & 1.79 & 95.67 & -0.19 & 7.73 & 20.10 & -26.45 & -0.41 & 8.45 \\
\hline 18 & 6.47 & 1.38 & 94.97 & -0.20 & 8.15 & 20.10 & -24.87 & -0.38 & 8.05 \\
\hline 19 & 5.04 & 0.73 & 94.83 & -0.23 & 8.24 & 20.10 & -22.71 & -0.35 & 7.54 \\
\hline 20 & 5.13 & 2.33 & 94.90 & -0.15 & 8.19 & 20.10 & -24.49 & -0.38 & 7.96 \\
\hline 21 & 4.84 & 4.53 & 94.75 & -0.05 & 8.28 & 20.10 & -26.45 & -0.41 & 8.45 \\
\hline 22 & 6.29 & 6.75 & 94.77 & 0.06 & 8.27 & 20.10 & -30.24 & -0.47 & 9.52 \\
\hline 23 & 5.40 & 8.68 & 94.96 & 0.15 & 8.15 & 20.10 & -31.45 & -0.48 & 9.89 \\
\hline 24 & 8.54 & 10.90 & 94.96 & 0.26 & 8.16 & 20.10 & -36.91 & -0.57 & 11.75 \\
\hline \multicolumn{8}{|c|}{ Average } & -0.61 & 13.36 \\
\hline
\end{tabular}


Table 33. Calculation of comfort indices PMV and PPD cement Room.

\begin{tabular}{|c|c|c|c|c|c|c|c|c|c|}
\hline \multirow{2}{*}{ Time } & \multicolumn{9}{|c|}{ Cement room } \\
\hline & $E_{r y}$ & $E_{c v}$ & $E_{p s}$ & $E_{c r}$ & $E_{h r}$ & $E_{\text {sd }}$ & $Q$ & $P M V$ & $P P D$ \\
\hline 1 & 6.52 & 11.53 & 94.92 & 0.29 & 8.18 & 20.10 & -35.53 & -0.55 & 11.25 \\
\hline 2 & 11.37 & 13.76 & 95.06 & 0.40 & 8.10 & 20.10 & -42.79 & -0.66 & 14.10 \\
\hline 3 & 14.75 & 15.34 & 94.94 & 0.48 & 8.17 & 20.10 & -47.77 & -0.73 & 16.37 \\
\hline 4 & 20.15 & 16.30 & 95.01 & 0.53 & 8.12 & 20.10 & -54.20 & -0.83 & 19.67 \\
\hline 5 & 21.81 & 16.30 & 95.01 & 0.53 & 8.12 & 20.10 & -55.86 & -0.86 & 20.59 \\
\hline 6 & 25.45 & 15.67 & 95.06 & 0.50 & 8.10 & 20.10 & -58.86 & -0.91 & 22.32 \\
\hline 7 & 28.68 & 11.71 & 96.45 & 0.29 & 7.26 & 20.10 & -58.50 & -0.90 & 22.10 \\
\hline 8 & 30.00 & 12.34 & 96.39 & 0.32 & 7.30 & 20.10 & -60.45 & -0.93 & 23.27 \\
\hline 9 & 27.03 & 11.71 & 96.45 & 0.29 & 7.26 & 20.10 & -56.84 & -0.87 & 21.14 \\
\hline 10 & 24.33 & 10.44 & 96.42 & 0.23 & 7.28 & 20.10 & -52.80 & -0.81 & 18.91 \\
\hline 11 & 19.95 & 9.17 & 96.38 & 0.17 & 7.30 & 20.10 & -47.07 & -0.72 & 16.04 \\
\hline 12 & 16.59 & 7.60 & 96.53 & 0.09 & 7.21 & 20.10 & -42.12 & -0.65 & 13.82 \\
\hline 13 & 13.16 & 6.01 & 96.52 & 0.02 & 7.22 & 20.10 & -37.03 & -0.57 & 11.80 \\
\hline 14 & 8.02 & 4.43 & 96.51 & -0.06 & 7.23 & 20.10 & -30.21 & -0.46 & 9.51 \\
\hline 15 & 4.52 & 2.05 & 97.84 & -0.19 & 6.42 & 20.10 & -24.75 & -0.38 & 8.02 \\
\hline 16 & 1.43 & 0.64 & 99.34 & -0.26 & 5.52 & 20.10 & -20.77 & -0.32 & 7.12 \\
\hline 17 & -0.02 & -2.34 & 95.63 & -0.39 & 7.75 & 20.10 & -14.73 & -0.23 & 6.07 \\
\hline 18 & -4.38 & -2.75 & 94.88 & -0.40 & 8.21 & 20.10 & -9.65 & -0.15 & 5.46 \\
\hline 19 & -5.08 & -3.06 & 94.90 & -0.42 & 8.19 & 20.10 & -8.63 & -0.13 & 5.37 \\
\hline 20 & -6.06 & -1.18 & 94.77 & -0.32 & 8.27 & 20.10 & -9.57 & -0.15 & 5.45 \\
\hline 21 & -4.57 & 1.05 & 94.81 & -0.22 & 8.25 & 20.10 & -13.42 & -0.21 & 5.88 \\
\hline 22 & -3.40 & 3.90 & 94.79 & -0.08 & 8.26 & 20.10 & -17.56 & -0.27 & 6.52 \\
\hline 23 & -1.88 & 6.14 & 94.98 & 0.03 & 8.14 & 20.10 & -21.52 & -0.33 & 7.28 \\
\hline 24 & 1.99 & 8.68 & 94.96 & 0.15 & 8.15 & 20.10 & -28.04 & -0.43 & 8.88 \\
\hline & & & & & & & & -0.54 & 12.79 \\
\hline
\end{tabular}

for the shade between two architectural proposals, one leading to slightly uncomfortable atmosphere and the other very uncomfortable atmospheres. The distinction is made on the footnote that establishes quality deviations at a given time on the sequence of study. Even a short study sequence provides a series of notes that can be considered as such because it would become difficult to read, which brings us to use parameter synthesizing these results as the average of those notes whose quality index $q$ will result.

The results of this calculation are shown in the following Table 34.

The results show us that whether we are in a building or land in a cement building for this hot day in April, we have a quality index $q$ such that: $80 \%<q<90 \%$. Depending on the scale of Figure 8, the two habitats are considered good thermal quality.

It is important to note that the number $\mathrm{q}$ is not a comfort index [19]. It characterizes the building in relation to the thermal fields that are created during the climatic sequence and the reaction of the user. It is not intended to make finely account statements comfort of a user, but rather to give an image a posteriori the conditions under which it perceived the atmosphere. The indices comfort them and have to assess the physiological reactions to environmental factors.

In conclusion, we can say that although the PPD in the land habitat is slightly higher than that in the cement housing, land habitat has almost the same thermal performance as the cement and with habitat very good 
Table 34. Calculation notes of comfort and quality of local heat.

\begin{tabular}{|c|c|c|c|c|c|c|}
\hline \multirow{2}{*}{ Time } & \multicolumn{3}{|c|}{ Ground room } & \multicolumn{3}{|c|}{ Cement room } \\
\hline & $P M V$ & $P P D$ & $n$ & PMV & PPD & $n$ \\
\hline 1 & -0.65 & 13.91 & 86.09 & -0.55 & 11.25 & 88.75 \\
\hline 2 & -0.71 & 15.75 & 84.25 & -0.66 & 14.10 & 85.90 \\
\hline 3 & -0.76 & 17.19 & 82.81 & -0.73 & 16.37 & 83.63 \\
\hline 4 & -0.80 & 18.61 & 81.39 & -0.83 & 19.67 & 80.33 \\
\hline 5 & -0.82 & 19.27 & 80.73 & -0.86 & 20.59 & 79.41 \\
\hline 6 & -0.83 & 19.46 & 80.54 & -0.91 & 22.32 & 77.68 \\
\hline 7 & -0.80 & 18.53 & 81.47 & -0.90 & 22.10 & 77.90 \\
\hline 8 & -0.81 & 18.76 & 81.24 & -0.93 & 23.27 & 76.73 \\
\hline 9 & -0.79 & 18.24 & 81.76 & -0.87 & 21.14 & 78.86 \\
\hline 10 & -0.72 & 16.04 & 83.96 & -0.81 & 18.91 & 81.09 \\
\hline 11 & -0.69 & 15.12 & 84.88 & -0.72 & 16.04 & 83.96 \\
\hline 12 & -0.64 & 13.53 & 86.47 & -0.65 & 13.82 & 86.18 \\
\hline 13 & -0.62 & 12.95 & 87.05 & -0.57 & 11.80 & 88.20 \\
\hline 14 & -0.56 & 11.54 & 88.46 & -0.46 & 9.51 & 90.49 \\
\hline 15 & -0.50 & 10.16 & 89.84 & -0.38 & 8.02 & 91.98 \\
\hline 16 & -0.48 & 9.88 & 90.12 & -0.32 & 7.12 & 92.88 \\
\hline 17 & -0.41 & 8.45 & 91.55 & -0.23 & 6.07 & 93.93 \\
\hline 18 & -0.38 & 8.05 & 91.95 & -0.15 & 5.46 & 94.54 \\
\hline 19 & -0.35 & 7.54 & 92.46 & -0.13 & 5.37 & 94.63 \\
\hline 20 & -0.38 & 7.96 & 92.04 & -0.15 & 5.45 & 94.55 \\
\hline 21 & -0.41 & 8.45 & 91.55 & -0.21 & 5.88 & 94.12 \\
\hline 22 & -0.47 & 9.52 & 90.48 & -0.27 & 6.52 & 93.48 \\
\hline 23 & -0.48 & 9.89 & 90.11 & -0.33 & 7.28 & 92.72 \\
\hline 24 & -0.57 & 11.75 & 88.25 & -0.43 & 8.88 & 91.12 \\
\hline$q_{j}$ & & 86.64 & & & 87.21 & \\
\hline
\end{tabular}

architectural design we can improve thermal comfort.

\section{Conclusions}

The study of bioclimatic comfort and thermal simulation has enabled the assessment of the thermal quality of ground adobe mixed with straw. The simulation analysis by the software CoDyBa confirmed the interesting behavior of earth materials. Fanger's model is a method of approach in terms of habitat comfort, supports the conclusion that the earth mixed with straw is a good thermal performance material.

Despite all the attention on building envelopes of land adobes habitat mixed with straw, the better quality thermal comfort can be achieved by taking into account the architectural parameters.

\section{References}

[1] DREM (Resource Directorate of Water and Meteorology) (1994) Weather Data of Chad. Several Fiches. N’Djaména (CHAD). From 1984-1993.

[2] Cabot, J., Bouquet, C., et al. (1972) ATLAS Practice CHAD. Insh. 77 p. 
[3] AFME (1989) Analyse climatique du site. Collection. Cahier pédagogique: Thermique et Architecture. 109 p.

[4] Dong, Q.F., Yu, L., Lu, H.J., Hong, Z. and Chen, Y.R. (2010) Design of Building Monitoring Systems Based on Wireless Sensor Networks. Wireless Sensor Network, 2, 703-709. http://dx.doi.org/10.4236/wsn.2010.29085

[5] Sigma Consultants, ERGM (1988) Habitats climatiques. Programme Interministériel Rexcoop, République Française. Bulletin.

[6] Favata, C.A., Christensen, D.R., Thompson, R., McKeown, K.A. and Hanselman, J.A. (2015) Evaluation of a Modified Habitat Suitability Index Model for Eastern Brook Trout: Implications for Efficient Habitat Assessment. Journal of Student Research, 4, 90-98.

[7] Gandemer, J. and Guyot, A. (1976) Intégration du phénomène vent dans la conception du milieu bâti. C.S.T.B: Science des bâtiments, Nantes.

[8] Jones, L.A., Muhlfeld, C.C., Marshall, L.A., McGlynn, B.L. and Kershner, J.L. (2013) Estimating Thermal Regimes of Bull Trout and Assessing the Potential Effects of Climate Warming on Critical Habitats. River Research and Applications, 30, 204-216.

[9] Ahmat-Charfadine, M. (2002) Experimental Study of Thermo Physical Properties of Local Materials Chad. Application to Thermal Comfort of Habitat. Thesis, Claude Bernard University Lyon 1, Lyon, 187 p.

[10] Mahamat, A.-C., Barka, M., Tahir, A.M., Soultan, M., Gaye, S. and Beye, A.C. (2015) Thermo-Physical Properties of Local Materials Used in the Construction of Chad. American Journal of Civil Engineering, 3, 140-149.

[11] Fanger, P.O. (1973) Thermal Comfort. McGraw-Hill Book Company, New York, 224 p.

[12] Norme AFNOR NFX 35-203 (1981) Détermination des indices PMV et PPD et spécifications des conditions de confort thermique. Association Française de Normalisation.

[13] Ashrae Handbook Fundamentals (1985) Physiological Principal, Comfort and Heat.

[14] Kim, G.-H. (2013) A New Platform for Building Researchers. Journal of Building Construction and Planning Research, 1, 25-26. http://dx.doi.org/10.4236/jbcpr.2013.12004

[15] Thellier, F., Cordier, A., Mouchoux, F. and Serin, G. (1989) Analyse des paramètres de confort thermique par modélisation d'un individu dans son habitat. Chauffage, Ventilation, Conditionnement, 65, 27-32.

[16] Depecker, P. (1986) Maîtrise des ambiances habitées et repérage de la qualité thermique. Annales de l'ITBP. Série: théories et méthodes de calcul 283. Mars-Avril 1986, Paris, No. 443, 49-64.

[17] Norme ISO 7730 (1984) Spécification des conditions de confort thermique. Organisation Internationale de Normalisation, Genève.

[18] Givoni, B. (1978) L’homme, l’architecture et le climat. Editions du moniteur, Paris, 461 p.

[19] Odunfa, K.M., Ojo, T.O., Odunfa, V.O. and Ohunakin, O.S. (2015) Energy Efficiency in Building: Case of Buildings at the University of Ibadan, Nigeria. Journal of Building Construction and Planning Research, 3, 18-26. http://dx.doi.org/10.4236/jbcpr.2015.31003 